\title{
A Review on JPEG2000 Image Compression
}

\author{
Raman Maini ${ }^{1}$ and Suruchi Mehra ${ }^{2}$ \\ ${ }^{1}$ Associate Professor, University College of Engineering,Punjabi University \\ Patiala, Punjab, India - 147002 \\ ${ }^{2}$ Research Scholar, University College of Engineering, Punjabi University \\ Patiala, Punjab, India - 147002
}

\begin{abstract}
Image compression requires higher performance as well as new features to address the need in the specific area of still image encoding, a new standard is currently being developed, that is JPEG2000. The JPEG2000 standard provides a set of features that are of vital importance to many high-end and emerging applications. JPEG-2000 is an emerging standard for still image compression. This paper provides a brief history of the JPEG-2000 standardization process, an overview of the standard, and some description of the capabilities provided by the standard. In this paper we describe JPEG-2000 from the point of view of encoding.
\end{abstract}

Keywords: JPEG, colour image compression, source coding, subband coding, wavelet transform

\section{INTRODUCTION}

Since the mid-80s, members from the International Telecommunication Union (ITU) and the International Organisation for Standardisation (ISO) have been working together to establish a joint international standard for the compression of continuous-tone (multilevel) still images, both greyscale and colour. This effort has been known as JPEG, the Joint Photographic Experts Group. (The "joint" in JPEG refers to the collaboration between ITU and ISO).

[7]With the continual expansion of multimedia and Internet applications, the needs and requirements of the technologies used, grew and evolved. In March 1997 a new call for contributions were launched for the development of a new standard for the compression of still images[1,2], the JPEG2000 was intended to create a new image coding system for different types of still images (bi-level, greylevel, colour, multicomponent) with different characteristic. (natural images, scientific, medical, remote sensing, text, rendered graphics, etc) allowing different imaging models(client/server, real-time transmission, image library archival, limited buffer and bandwidth resources, etc) preferably within a unified system address efficiently or not address at all .To address this need in the specific area of still image encoding, a new standard is currently being developed, the JPEG2000. It is not only intended to provide rate-distortion and subjective image quality performance superior to existing standards, but also to provide functionality that current standards can either not addresses efficiently or not address at all.

The markets and applications better served by the JPEG2000 standard are Internet, colour facsimile, printing, scanning (consumer and pre-press), digital photography, remote sensing, mobile, medical imagery, digital libraries archives and E-commerce. Each application area imposes some requirements that the standard should fulfil (Requirements AHG, 1999). $[3,4]$ The main features that this standard possesses are: superior low bit-rate performance, continuoustone and bi-level compression, lossless and lossy compression, progressive transmission by pixel accuracy and resolution, random codestream access and processing, robustness to bit-errors.

In the present Letter the structure of the JPEG2000 standard is presented and performance comparisons are reported the aim of JPEG 2000 is not only improving compression performance over JPEG but also adding (or improving) features such as scalability and editability. In fact, JPEG 2000 's improvement in compression performance relative to the original JPEG standard is actually rather modest and should not ordinarily be the primary consideration for evaluating the design. Very low and very high compression rates are supported in JPEG 2000. In fact, the graceful ability of the design to handle a very large range of effective bit rates is one of the strengths of JPEG 2000. For example, to reduce the number of bits for a picture below a certain amount, the advisable thing to do with the first JPEG standard is to reduce the resolution of the input image before encoding it. That's unnecessary when using JPEG 2000, because JPEG 2000 already does this automatically through its multiresolution decomposition structure. The following sections describe the architecture of JPEG 2000.

\section{ARCHITECTURE}

Initially, images have to be transformed from the RGB color space to another color space, leading to three components that are handled separately. There are two possible choices:

Irreversible Color Transform (ICT) uses the well known $\mathrm{YC}_{\mathrm{B}} \mathrm{C}_{\mathrm{R}}$ color space. It is called "irreversible" because it has to be implemented in floating or fix-point and causes round-off errors.

Reversible Color Transform (RCT) uses a modified YUV color space that does not introduce quantization errors, so it is fully reversible. Proper implementation of the RCT requires that numbers are rounded as specified that cannot be expressed exactly in matrix form. The transformation is:

$$
\begin{aligned}
& Y_{r}[(R+2 G+B) / 4] ; C_{b}=B-G ; C_{r}=R-G ; \\
& \text { and } \\
& G=Y-\left[\left(C_{b}+C_{r}\right) / 4\right] ; R=C_{r}+G ; B=C_{b}+G
\end{aligned}
$$

The chrominance components can be, but do not necessarily have to be, down-scaled in resolution; in fact, since the wavelet transformation already separates images into scales, downsampling is more effectively handled by dropping the finest wavelet scale. This step is calledmultiple component transformation in the JPEG 2000 language since its usage is not restricted to the RGB color model. 


\section{Tiling:}

After color transformation, the image is split into socalled tiles, rectangular regions of the image that are transformed and encoded separately. Tiles can be any size, and it is also possible to consider the whole image as one single tile. Once the size is chosen, all the tiles will have the same size (except optionally those on the right and bottom borders). Dividing the image into tiles is advantageous in that the decoder will need less memory to decode the image and it can opt to decode only selected tiles to achieve a partial decoding of the image [10]. The disadvantage of this approach is that the quality of the picture decreases due to a lower peak signal-to-noise ratio. Using many tiles can create a blocking effect similar to the older JPEG 1992 standard.

\subsection{Wavelet transform:}

Tile components are decomposed into different decomposition levels using a wavelet transform. These decomposition levels contain a number of subbands populated with coefficients that describe the horizontal and vertical spatial frequency characteristics of the original tile component planes. These tiles are then wavelet transformed to an arbitrary depth, in contrast to JPEG 1992 which uses an $8 \times 8$ block-size discrete cosine transform. JPEG 2000 uses two different wavelet transforms:

Irreversible: The CDF 9/7 wavelet transform.(figure 1 ) It is said to be "irreversible" because it introduces quantization noise that depends on the precision of the decoder.

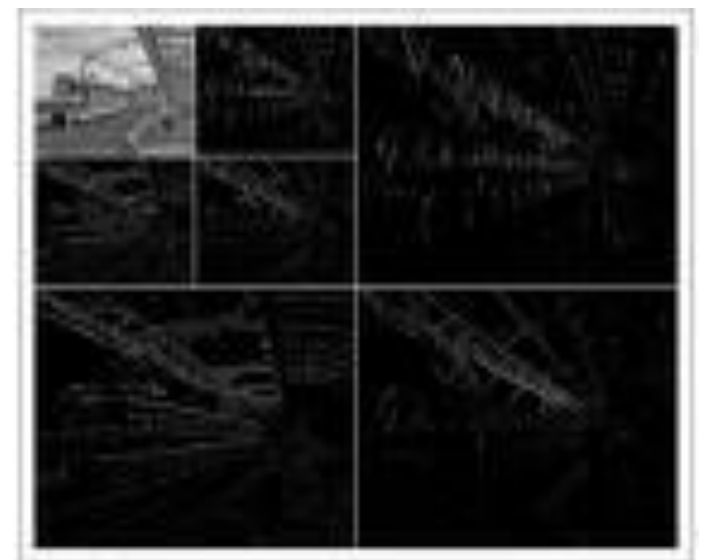

Figure 1:CDF9/7 wavelet transformation

The picture shows a 2 level wavelet transform. For the low-pass and high-pass filters, I have used the same of the JPEG2000, lossy transform, so that this is the wavelet that is used in the JPEG2000 format. In general, it is quite hard to show a wavelet transform properly, because of the different ranges of the different parts [5 6]

Reversible: A rounded version of the biorthogonal CDF 5/3 wavelet transform (figure2).
It uses only integer coefficients, so the output does not require rounding (quantization) and so it does not introduce any quantization noise. It is used in lossless coding.

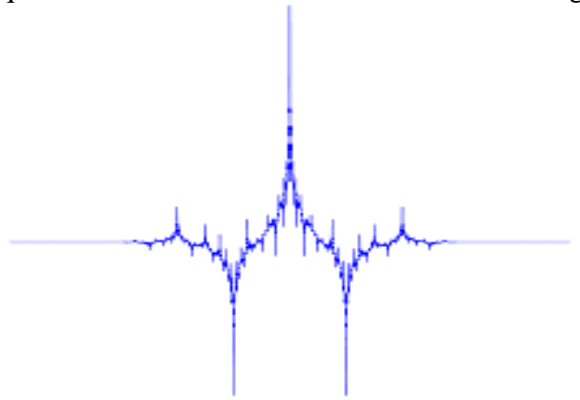

Figure 2: CDF 5/3 wavelet transformation

The wavelet transforms are implemented by the lifting scheme or by convolution.

For both ionmodes to be implemented, the signal should be first extended periodically as shown in Fig. 3. This periodic symmetric extension is used to ensure that for the filtering operations that take place at both boundaries of the signal, one signal sample exists and spatially corresponds to each coefficient of the filter mask. The number of additional samples required at the boundaries of the signal is therefore filter-length dependent (ISO/IEC, 2000).

\subsection{Quantization}

After the wavelet transform, the coefficients are scalarquantized to reduce the amount of bits to represent them, at the expense of a loss of quality. The output is a set of integer numbers which have to be encoded bit-by-bit. The parameter that can be changed to set the final Quality is the quantization step: [4] The greater the step, the greater is the compression and the loss of quality. With a quantization step that equals 1 , no quantization is performed (it is used in lossless compression).

Quantization operation is defined by the step size $\Delta b$, the step size is quite fixable, but there are a few restrictions imposed by the standard.

1. Reversible wavelets: when reversible wavelets are utilized in JPEG2000, uniform dead zone scalar quantization with a step size of $\Delta b=1$ must be used.

2. Irreversible wavelets: when irreversible wavelets are utilized in JPEG2000, the step size selection is restricted only by the signaling syntax itself

$$
\Delta_{b}=2^{-\varepsilon_{b}}\left(1+\frac{\mu_{b}}{2^{11}}\right)
$$




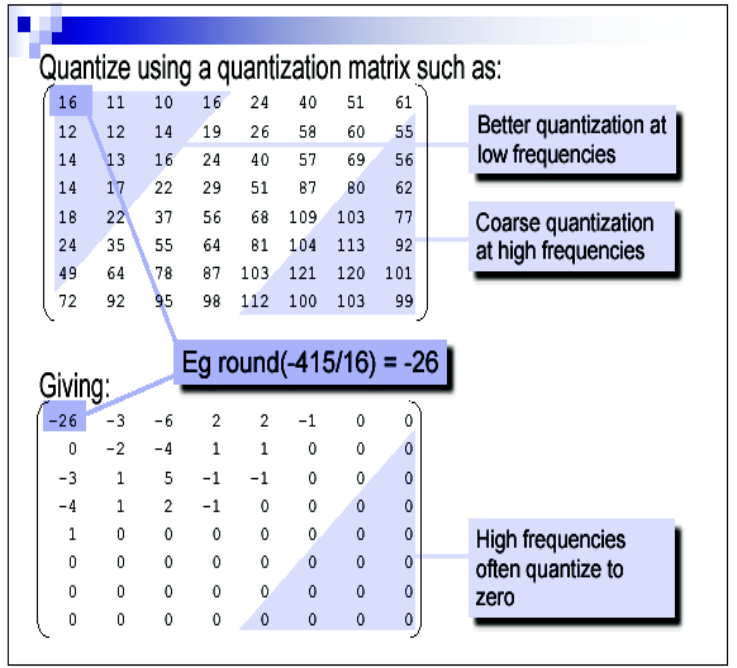

Figure 3

An optimal strategy to select quantization step size for each sub band is:

$$
\Delta_{b}=\Delta \cdot \sqrt{\frac{1}{G_{b}}} \quad G_{b} \approx 2^{2 d_{b}}
$$

$d_{b}$ denotes the level index of DWT for sub band b.

Two kinds of quantization in JPEG2000:

1. The Direct truncation

\begin{tabular}{|l|l|l|l|}
\hline $2^{\wedge} \mathbf{n}$ & $2^{\wedge} \mathbf{n}$ & \multirow{2}{*}{$2^{2_{n}}$} & \multirow{2}{*}{$2^{\wedge} \mathbf{n}$} \\
\hline $2^{2_{n}}$ & $2^{\wedge} \mathbf{n}$ & \\
\hline $2^{2^{\wedge} \mathbf{n}}$ & $2^{2^{\wedge} \mathbf{n}}$ & \\
\hline \multicolumn{2}{|l|}{} & $2^{2^{\wedge} \mathbf{n}}$ \\
& & \\
\hline
\end{tabular}

\section{The variable truncation}

\begin{tabular}{|l|l|l|l|}
\hline $2^{\wedge} \mathbf{n}$ & $2^{\wedge} \mathbf{n}$ & \multirow{2}{*}{$2^{\wedge}(\mathrm{n}+1)$} & \multirow{2}{*}{$2^{\wedge}(\mathrm{n}+1)$} \\
\hline $2^{\wedge} \mathrm{n}$ & $2^{\wedge} \mathrm{n}$ & & \\
\hline $2^{\wedge}(\mathrm{n}+1)$ & $2^{\wedge}(\mathrm{n}+1)$ & \\
\hline & & \\
$22^{\wedge}(\mathrm{n}+1)$ & & \\
& & \\
& & \\
\hline
\end{tabular}

\subsection{Entropy coding:}

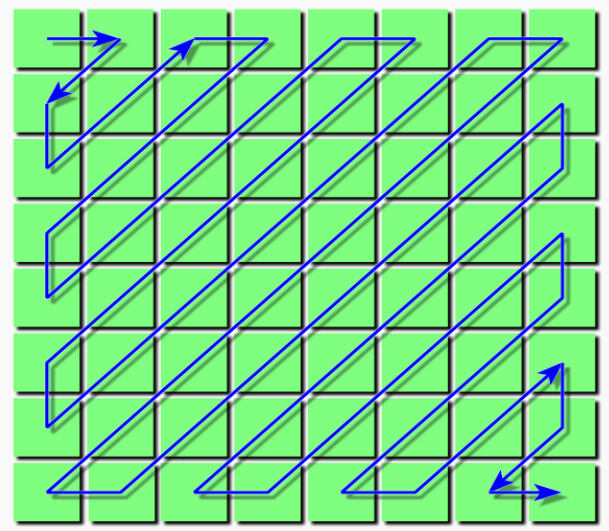

Figure 4

Zigzag ordering of JPEG image components

Components in a "zigzag" order employing run-length encoding (RLE) algorithm that groups similar frequencies together, inserting length coding zeros, and then using Huffman coding on what is left.

The JPEG standard also allows, but does not require decoders to support, the use of arithmetic coding, which is mathematically superior to Huffman coding. However, this feature has rarely been used as it was historically covered by patents requiring royalty-bearing licenses, and because it is slower to encode and decode compared to Huffman coding. Arithmetic coding typically makes files about 5-7\% smaller.

The previous quantized DC coefficient is used to predict the current quantized DC coefficient. The difference between the two is encoded rather than the actual value. The encoding of the 63 quantized AC coefficients does not use such prediction differencing.

The zigzag sequence for quantized coefficients are shown below. (The format shown is just for ease of understanding/viewing.)Entropy coding is a special form of lossless data compression. It involves arranging the image

$$
\begin{array}{lllllllll}
-26 & & & & & & & \\
-3 & 0 & & & & & & \\
-3 & -2 & -6 & & & & & \\
2 & -4 & 1 & -3 & & & & \\
1 & 1 & 5 & 1 & 2 & & & \\
-1 & 1 & -1 & 2 & 0 & 0 & & \\
0 & 0 & 0 & -1 & -1 & 0 & 0 & \\
0 & 0 & 0 & 0 & 0 & 0 & 0 & 0 \\
0 & 0 & 0 & 0 & 0 & 0 & 0 & \\
0 & 0 & 0 & 0 & 0 & 0 & & \\
0 & 0 & 0 & 0 & 0 & & & \\
0 & 0 & 0 & 0 & & & & \\
0 & 0 & 0 & & & & & \\
0 & 0 & & & & & & \\
0 & & & & & & &
\end{array}
$$


If the $\mathrm{i}$-th block is represented by $\mathrm{Bi}$ and positions within each block are represented by $(p, q)$ where $p=0,1, \ldots, 7$ and $q=0,1$, ..., 7 , then any coefficient in the DCT image can be represented as $\operatorname{Bi}(\mathrm{p}, \mathrm{q})$. Thus, in the above scheme, the order of encoding pixels (for the i-th block) is $\operatorname{Bi}(0,0), \operatorname{Bi}(0,1), \operatorname{Bi}(1,0), \operatorname{Bi}(2,0)$, $\operatorname{Bi}(1,1), \operatorname{Bi}(0,2), \operatorname{Bi}(0,3), \operatorname{Bi}(1,2)$ and so on.

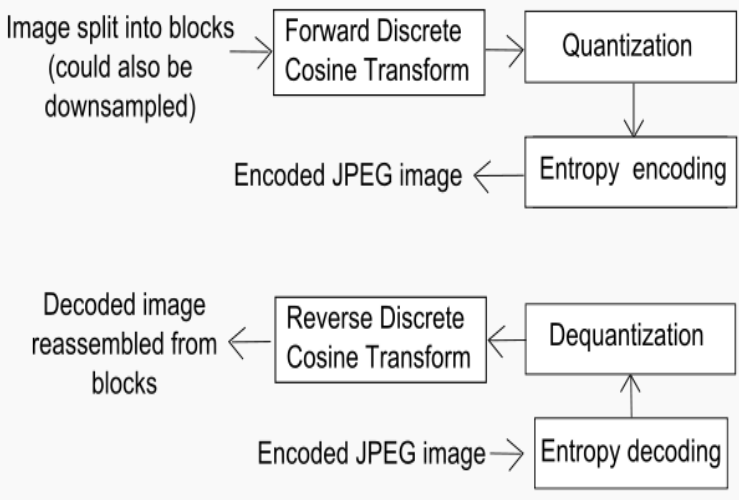

Baseline sequential JPEG encoding and decoding processes

This encoding mode is called baseline sequential encoding. Baseline JPEG also supports progressive encoding. While sequential encoding encodes coefficients of a single block at a time (in a zigzag manner), progressive encoding encodes similar-positioned coefficients of all blocks in one go, followed by the next positioned coefficients of all blocks, and so on. So, if the image is divided into $\mathrm{N} 8 \times 8$ blocks $\{\mathrm{B} 0, \mathrm{~B} 1, \mathrm{~B} 2, \ldots, \mathrm{Bn}-1\}$, then progressive encoding encodes $\operatorname{Bi}(0,0)$ for all blocks, i.e., for all $\mathrm{i}=0,1,2, \ldots, \mathrm{N}-1$. This is followed by encoding $\operatorname{Bi}(0,1)$ coefficient of all blocks, followed by $\mathrm{Bi}(1,0)$-th coefficient of all blocks, then $\mathrm{Bi}(2,0)$-th coefficient of all blocks, and so on. It should be noted here that once all similar-positioned coefficients have been encoded, the next position to be encoded is the one occurring next in the zigzag traversal as indicated in the figure above. It has been found that Baseline Progressive JPEG encoding usually gives better compression as compared to Baseline Sequential JPEG due to the ability to use different Huffman tables (see below) tailored for different frequencies on each "scan" or "pass" (which includes similar-positioned coefficients), though the difference is not too large[7].

In the rest of the article, it is assumed that the coefficient pattern generated is due to sequential mode.

In order to encode the above generated coefficient pattern, JPEG uses Huffman encoding. JPEG has a special Huffman code word for ending the sequence prematurely when the remaining coefficients are zero.

Using this special code word: "EOB", the sequence becomes:

$\begin{array}{lllllll}-26 & & & & & \\ -3 & 0 & & & & \\ -3 & -2 & -6 & & & \\ 2 & -4 & 1 & -3 & & \\ 1 & 1 & 5 & 1 & 2 & \\ -1 & 1 & -1 & 2 & 0 & 0 \\ 0 & 0 & 0 & -1 & -1 & \text { EOB }\end{array}$

JPEG's other code words represent combinations of (a) the number of significant bits of a coefficient, including sign, and (b) the number of consecutive zero coefficients that precede it. (Once you know how many bits to expect, it takes 1 bit to represent the choices $\{-1,+1\}, 2$ bits to represent the choices $\{-$ $3,-2,+2,+3\}$, and so forth.) In our example block, most of the quantized coefficients are small numbers that are not preceded immediately by a zero coefficient. These more-frequent cases will be represented by shorter code words. [8,9]

The JPEG standard provides general-purpose Huffman tables; encoders may also choose to generate Huffman tables optimized for the actual frequency distributions in images being encoded.

\section{PERFORMANCE}

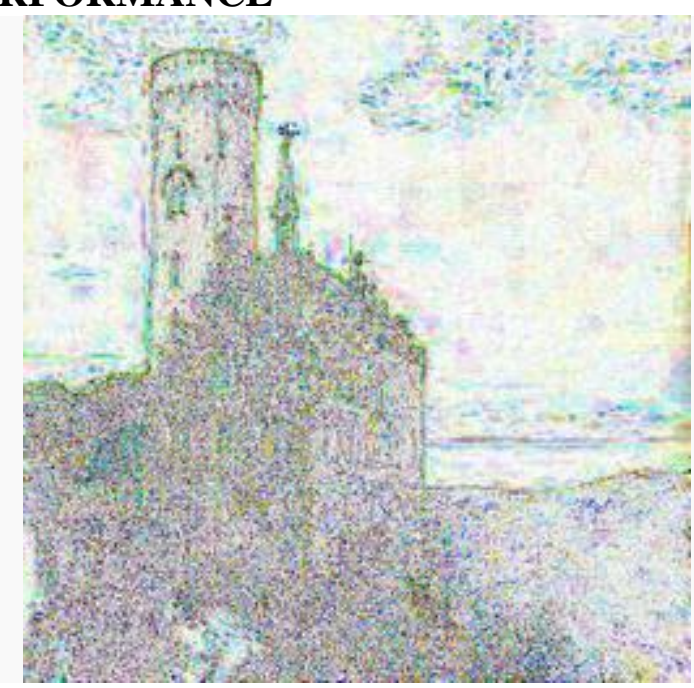

Figure 5

This Figure 5shows the (accentuated) difference between an image saved as JPEG 2000 (quality 50\%) and the original [5].

Compared to the previous JPEG standard, JPEG 2000 delivers a typical compression gain in the range of $20 \%$, depending on the image characteristics. Higher-resolution images tend to benefit more, where JPEG-2000's spatial-redundancy prediction can contribute more to the compression process. In very low-bitrate applications, [11, 12] studies have shown JPEG 2000 to be outperformed $^{[21]}$ by the intra-frame coding mode of H.264. Good applications for JPEG 2000 are large images, images with lowcontrast edges - e.g., medical images.

\section{CONCLUSION}

A major advantage of using a JPEG 2000 hardware solution is lower latency than any other compression scheme., It offers numerous benefits over current compression methods, including the ability to do both lossless and lossy compression, the ability to obtain higher image quality and higher compression ratios, and the ability to view the same file at multiple resolutions. It also allows one area of the image to be examined more closely using its region of interest capability. JPEG 2000 uses wavelet technology to compress images, and result in images being compressed more efficiently and with less introduced error than previous methods.

There are many applications where JPEG 2000 can be used, including the Internet, medical imaging, and digital photography 


\section{REFERENCES}

[1] Ahmed, N., T. Natarajan, and K. R. Rao. 1974. On image processing and a discrete cosine transform. IEEE Transactions on Computers C-23(1): 90-93.

[2] A.N. Skodra, C.A. Christopoulos, T. Ebrahimi, «JPEG2000, the upcoming still image compression standard », Proceeding of the $11^{\text {th }}$ Portuguese Conference on Pattern recognition, Porto, Portugal, May 11th-12th, pp. 359-366, 2000

[3] Introduction to Data Compression, K. Sayood, Morgan Kauffman, Second Edition, 2000. (Primary).

[4]. ISO/IEC, ISO/IEC 14495-1:1999: Information technologyLossless and near-lossless compression of continuous-tone still.

[5] ISO/IEC, ISO/IEC 14496-2:1999: Information technologyCoding of audio-visual objects - Part 2: Visual, December 1999.

[6] ISO/IEC JTC1/SC29/WG1, Call for contributions for JPEG 2000(JTC 1.29.14, 15444): Image Coding System, WG1 N 505, March 1997, http://www.jpeg.org/public/wg1n505.pdf.
[7]. ISO/IEC JTC 1/SC 29/WG 1, ISO/IEC FCD 15444-1: Information technology - JPEG 2000 image coding system: Core coding system [WG 1 N 1646]

[8] Jain, A. K. 1989. Fundamentals of digital image processing., Prentice Hall: Englewood Cliffs, NJ Wallace, G. 1991. The JPEG still picture compression standard,Communications of the ACM 34(4): 30-44.

[9] Shannon, C. E., and W. Weaver. 1949. The mathematical theory of communication. Urbana: University of Illinois Press March 2000, http://www.jpeg.org/FCD15444-1.htm.

[10] Wallace, G. 1991. The JPEG still picture compression standard. Communications of the ACM 34(4): 30-44.

[11]. William B. Pennebaker and Joan L. Mitchell, JPEG: Still Image Data Compression Standard, Van Nostrand Reinhold, New York, 1992. Images: Baseline, Dec. 1999.

[12]Watson, A. B. 1993. DCT quantization matrices visually optimized for individual images. Proceedings of the SPIE 1913:202-216. (Human Vision, Visual Processing, and Digital Display IV. Rogowitz ed. SPIE. Bellingham,WA). 